\title{
Pulsed Transient Tissue Conductivity Measurement
}

\author{
Robert B. Szlavik and Hubert De Bruin \\ Department of Electrical and Computer Engineering and Medicine \\ McMaster University, Hamilton, Ontario, Canada, L8N 3Z5
}

\begin{abstract}
We present an alternative technique for obtaining tissue conductivity as a function of frequency. Pulsed transient tissue impedance measurement differs significantly from other commonly used swept frequency techniques in that the conductivity of a tissue sample can be obtained from a voltage transient response to a current pulse. By obtaining the conductivity across a band of frequencies from a transient measurement, the necessity for direct conductivity measurements at each frequency point is eliminated. Calibration and verification of the technique was carried out by measuring the impedance magnitude and phase of simple RC circuit combinations. We present conductivity measurements taken from in vitro poultry skeletal muscle tissue specimens. Measurements from fresh animal tissues are currently being obtained.
\end{abstract}

\section{Introduction}

We present an alternative technique for obtaining the conductivity magnitude and phase of tissue as a function of frequency. The method is based on the electrical transient response of a tissue specimen to a current pulse. This technique is an application of a method first proposed by Teorell [1] which he applied to equivalent circuit models of tissue. Our investigations suggest that this method can be applied to tissue conductivity measurements and provides a viable alternative to other techniques reported in the literature.

The method incorporates the standard four electrode configuration, shown in Fig. 1, , and used by other authors to measure tissue conductivity [2]. The traditional method used to measure tissue conductivity as a function of frequency is based on a swept frequency technique. In the swept frequency technique, the tissue is stimulated with a sinusoidal current source. The frequency of the sinusoid is swept in increments and the conductivity is measured at each frequency step [2]. By stimulating the tissue specimen with a transient current pulse and applying Fourier analysis techniques to the response potential measured between the two inner electrodes, the conductivity frequency response can be calculated from the transient response and from measurements of the excitation current time domain waveform.

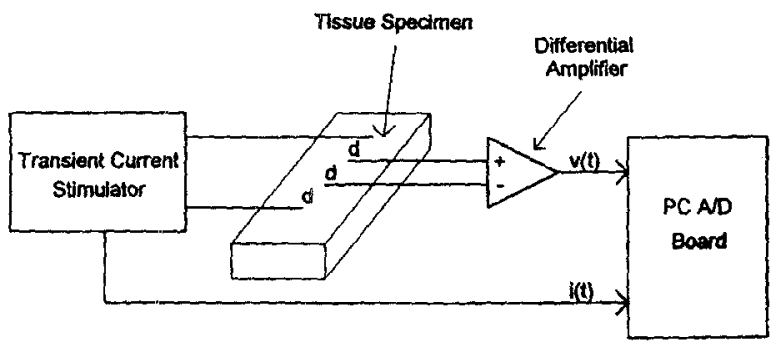

Figure 1. Block diagram illustrating the major components of the pulsed transient tissue conductivity experiment. A transient current stimulator is used to generate a current pulse. The resulting potential measured between the two voltage sensing electrodes is amplified and the signal recorded using an anslog to digital conversion board in a PC. In addition, the stimulation current waveform is also sampled using the same A/D board. The interelectrode distance shown is $\mathrm{d}$.

To calibrate and verify the pulse transient technique, we used simple RC circuit combinations. In addition, we tested the system with poultry and pork skeletal muscle tissue and measured the anisotropic conductivity magnitude in the directions parallel and perpendicular to the muscle fibers. 


\section{Theory}

\section{A. Parallel RC Circuit Impedance}

For a parallel RC circuit, the impedance $Z(f)$, as a function of frequency, is shown in (1).

$$
Z(f)=\frac{R}{1+j 2 \pi f R C}
$$

This equation is derived from the parallel impedance relationship where the overall impedance is represented as a complex number.

\section{B. Anisotropic Conductivity of Skeletal Muscle Tissue}

Skeletal muscle tissue is anisotropic because the conductivity measured in the direction parallel to the muscle fibers will be greater than the conductivity in the perpendicular direction [3]. A formula that relates the measured potential between the inner two electrodes and the anisotropic conductivities, using the four electrode configuration, has been derived by Rush [4] and used by others [2] in tissue conductivity measurements. We re-write these formulas with a modification where the current variable $I(f)$ and the measured voltages in the high conductivity and low conductivity direction $V_{h}(f)$ and $V_{l}(f)$ respectively are explicitly shown as functions of frequency. The variables $\phi_{h}(f)$ and $\phi_{l}(f)$ represent the phase shift between the voltage and current for the high conductivity and low conductivity directions respectively. In our formulation (2), these quantities represent the Fourier Transform of the measured time domain functions.

$$
\begin{gathered}
\sigma(f)=\frac{I(f)}{2 \pi d\left(V_{k}(f)\right.} \exp \left[j \phi_{h}(f)\right] \\
\sigma_{h}(f)=\left[\frac{I(f)}{V_{t}(f)}\right]^{2} \frac{V_{h}(f)}{2 \pi d l(f)} \exp \left[j\left(2 \Phi_{h}(f)-\Phi_{h}(f)\right)\right]
\end{gathered}
$$

The conductivity in the high and low directions, $\sigma_{h}(f)$ and $\sigma_{l}(f)$ respectively, are also written explicitly as functions of frequencies. Another important parameter in conductivity measurements is the inter-electrode spacing $d$ as shown in Fig. 1.

\section{Methodology}

A simplified block diagram of the instrumentation used in pulsed transient impedance measurements was illustrated in Fig. 1. An isolated current stimulator, Dogwood model
CMS1-200, was used to inject and remove current through the two outer current electrodes shown in Fig. 1. The current pulse width can be set in five incremental steps for pulse widths from $100 \mu \mathrm{s}$ to $2 \mathrm{~ms}$ and the amplitude of the excitation current pulse is adjustable from approximately 50 $\mu \mathrm{A}$ to $100 \mathrm{~mA}$.

For the measurements presented, pulse widths of $100 \mu \mathrm{s}$ were used. Theoretically it would be advantageous to use as narrow pulses in time as are practical since the energy content of these pulses would be relatively greater at higher frequencies which would allow for an improved broadband signal to noise ratio. In order to measure the current pulse as a function of time, a $20.3 \mathrm{kn}$ resistor was placed in series with each of the two current electrodes. The time waveform of the potential drop across this resistor was measured after a simple voltage follower stage constructed with an Analog Devices OP-27 low noise operational amplifier.

We measured the potential between the two inner electrodes using a National Semiconductor LM363D instrumentation amplifier with a gain of 100 . Both the signal from the instrumentation amplifier and the voltage follower were sampled at $300 \mathrm{kHz}$ using an A/D board, National Instruments AT-MIO-16E-1. A sampling program was implemented in LABVIEW and the sampled data was synchronously averaged and recorded using the application software that we developed.

\section{Results and Discussion}

The impedance magnitude and phase of several parallel RC circuits were obtained using the pulsed transient technique. Fig. 2. shows a measurement of the impedance magnitude and phase for a parallel $\mathrm{RC}$ circuit with a resistance of 100 $\Omega$ and a capacitance of $1.47 \mu \mathrm{F}$. Superimposed on this plot is the simulated impedance of this circuit calculated using PSPICE. These measurements were made with $100 \mu \mathrm{s}$ current pulses As would be expected, the impedance magnitude at low frequencies for this circuit tends to $100 \Omega$. At a frequency of $1.88 \mathrm{kHz}$, the impedance magnitude drops to $50 \mathrm{n}$, half of its low frequency value. The half value impedance frequency, obtained from the measured data, corresponds with the theoretically calculated value.

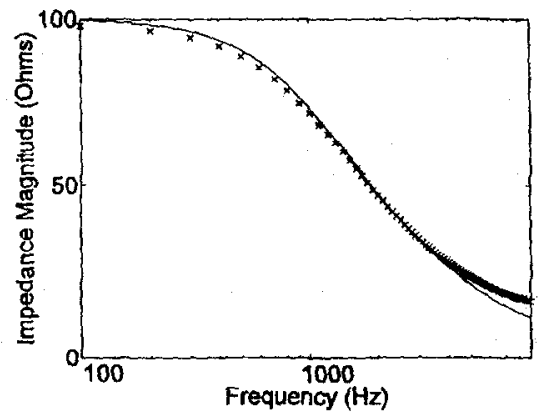




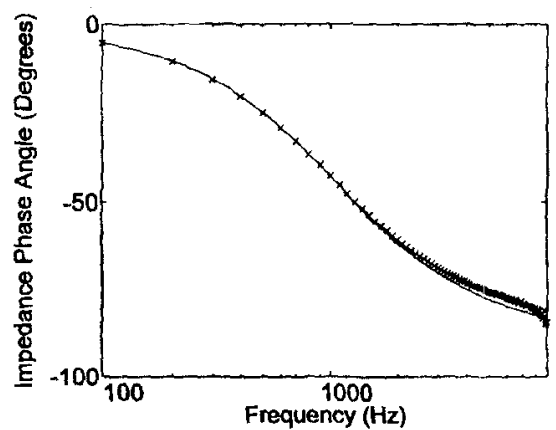

Figure 2. Measured impedance magnitude and phase of a parallel RC circuit combination with $R=100 \Omega$ and $C=1.47 \mu F$. A current pulse width of 100 us was used in this measurement. Experimental results are compared to theoretical impedance calculations from PSPICE. Simulated impedance using PSPICE - -, measured impedance - $x$.

In addition to the simple circuit tests mentioned above, in vitro measurements were made on pork and poultry skeletal muscle tissue. The formulas for the conductivity in (2) were applied to these measurements to obtain the frequency dependent curves shown in Fig. 3. of the conductivity magnitude and phase in the directions parallel and perpendicular to the muscle fibers. An inter-electrode spacing of $1 \mathrm{~mm} \pm 100 \mu \mathrm{m}$ was used for these measurements. Placement of the electrodes was made using a stereoscopic microscope and a glass reticle with $100 \mu \mathrm{m}$ divisions that was physically placed on the tissue specimen. Current pulses between $400 \mu \mathrm{A}$ and $500 \mu \mathrm{A}$ were used to make the measurements shown in Fig. 3.

As can be seen from Fig. 3., the conductivity parallel to the muscle fibers was greater across most of the band which is as expected. In general, the conductivity magnitudes that we obtained were greater than would be expected. These results are likely due to the fact that our measurements were not made on fresh animal tissue. An increase in tissue conductivity with time after death has been reported by other investigators [5]. The behavior of the phase response with frequency was consistent with observations made by Gielen et al. [2] for the bandwidth investigated. Our results confirm Gielen's observations of a positive phase shift in the conductance for the direction parallel to the muscle fibers and a negative phase shift for the perpendicular direction within the bandwidth investigated.

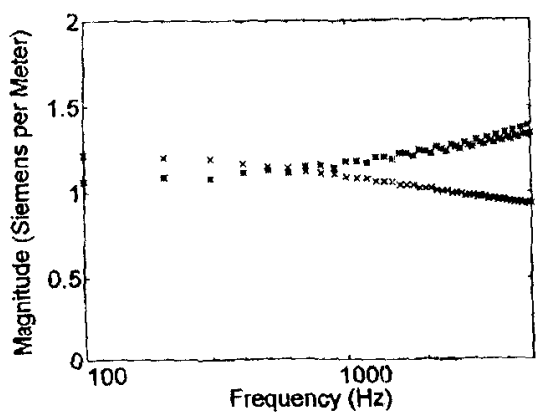

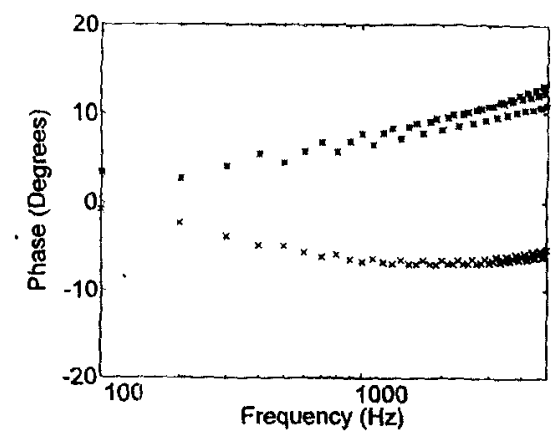

Figure 3. Conductivity magnitude and phase of poultry skeletal muscle tissue showing the conductivity in the directions paralfel and perpendicular to the muscle fibers. These measurements were made with an inter-electrode spacing of $\mathrm{d}=1 \mathrm{~mm} \pm 100 \mu \mathrm{m}$, a current pulse width of $100 \mu \mathrm{s}$ and a current pulse amplitude between $400 \mu \mathrm{A}$ and $500 \mu \mathrm{A}$. Parallel conductivity data $-\star$, perpendicular conductivity data $-x$.

\section{Conclusion}

We have presented an alternative method for obtaining the magnitude and phase of the conductivity as a function of frequency in tissue. A principle limitation of the technique is the bandwidth of the conductivity that is obtainable from these measurements. For the current pulse magnitudes that we have used in our experiments, bandwidth limitations are less than half of the inverse width of the current pulse. The bandwidth limitation can be overcome by using a more narrow current pulse to excite the tissue however limitations associated with the sampling hardware place a lower limit on the usable current pulse width. It was found that in most cases, digital filtering of the time domain waveforms was required.

This research was supported by a grant from the Natural Sciences and Engineering Research Council.

\section{References}

[1] T. Teorell, "Application of square wave analysis to bioelectric studies," Acta Physiol. Scand., vol. 12, pp. 235-254, 1947.

(2) F. L. H. Gielen, W. Wallinga-de Jonge and K. L. Boon, "Electrical conductivity of skeletal muscle tissue: experimental results from different muscles in vivo," Medical \& Biological Engineering \& Computing, vol. 22, pp. 569-577, 1984.

[3] F. L. H. Gielen, H, E. P. Cruts, B. A. Albers, K. L. Boon, W. Wallinga-de Jonge and H. B. K. Boom, Model of electrical conductivity of skeletal muscle based on tissue structure," Medical \& Biological Engineering \& Computing, vol. 24, pp. 3440, 1986.

[4] S. Rush, "Melhods of measuring the resistivities of anisotropic conducting media in situ," Joumal of Research of the National. Bureau of Standands, Section C, vol. 66C, no. 3, 1962

[5] L. A. Geddes and L. E. Baker, "The specific resistance of biological material - A compendium of data for the biomedical engineer and physiologist," Medical and Biological Engineering, vol. 5, pp. 271-293, 1967. 